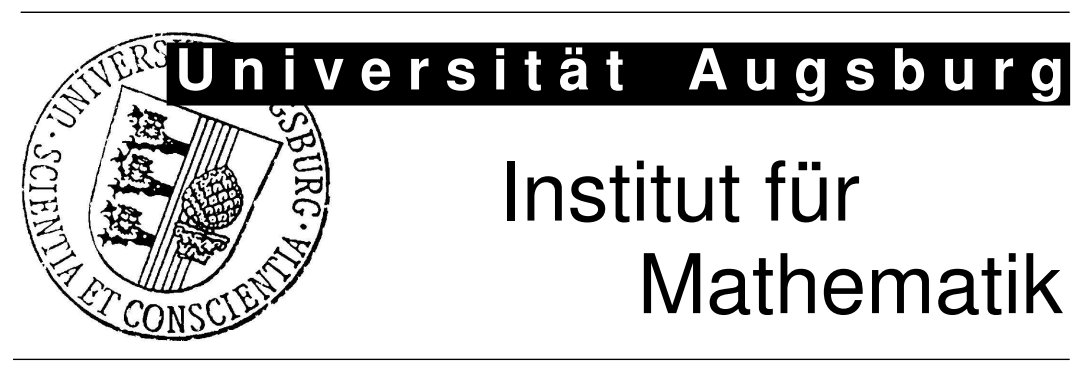

Martin Rasmussen, Peter Giesl

\title{
A Note on Almost Periodic Variational Equations
}




\section{Impressum:}

Herausgeber:

Institut für Mathematik

Universität Augsburg

86135 Augsburg

http://www . math. uni-augsburg.de/forschung/preprint/

ViSdP:

Martin Rasmussen

Institut für Mathematik

Universität Augsburg

86135 Augsburg

Preprint: Sämtliche Rechte verbleiben den Autoren (C) 2008 


\title{
A NOTE ON ALMOST PERIODIC VARIATIONAL EQUATIONS
}

\author{
PETER GIESL AND MARTIN RASMUSSEN
}

\begin{abstract}
The variational equation of a nonautonomous differential equation $\dot{x}=F(t, x), x \in \mathbb{R}^{N}$, along a solution $\mu$ is given by $\dot{x}=D_{x} F(t, \mu(t)) x$. We consider the question if the variational equation is almost periodic provided that the original equation is almost periodic by a discussion of the following problem: Is the derivative $D_{x} F$ almost periodic whenever $F$ is almost periodic? We give a negative answer in this paper, and the counterexample relies on an explicit construction of a scalar almost periodic function whose derivative is not almost periodic. Moreover, we provide a necessary and sufficient condition for the derivative $D_{x} F$ to be almost periodic.
\end{abstract}

\section{INTRODUCTION}

When studying the dynamical behavior of a nonautonomous differential equation $\dot{x}=F(t, x), x \in \mathbb{R}^{N}$, in the vicinity of a solution $\mu$, one usually first analyzes the linearization along this solution, which is given by the variational equation $\dot{x}=D_{x} F(t, \mu(t)) x$. It is not clear a priori if certain structures of the right-hand side carry over to the variational equation. For instance, if the right-hand side $F$ is periodic with respect to $t$, then obviously $D_{x} F$ is periodic, and thus, the variational equation along a periodic solution is also periodic. Does the same hold for almost periodic differential equations? This question arose in [GR], where almost periodicity of the variational equation was an extra assumption (cf. [GR, Theorem 4.1, 4.4]).

Before we discuss the case of almost periodic differential equations $\dot{x}=F(t, x)$, let us consider scalar functions $f: \mathbb{R} \rightarrow \mathbb{R}$. The derivative of a periodic function $f$ is obviously periodic, but is the derivative of an almost periodic function $f$ also almost periodic? One of the first theorems in many books on almost periodic functions states that the derivative is almost periodic if and only if it is uniformly continuous (see, e.g., [Fin74, Theorem 1.16] or [Cor68, Theorem 1.3, 1.8]). The theorem suggests that the derivative of an almost periodic function is not almost periodic in general, but to our best knowledge, a counterexample has not been provided in the literature yet. We will present the explicit construction of such a counterexample in Section 3.

This counterexample is then used in Section 4 to construct the right-hand side of an almost periodic differential equation $\dot{x}=F(t, x)$ such that $D_{x} F$ is not almost periodic. In Section 5 , we provide a necessary and sufficient condition for the derivative $D_{x} F$ to be almost periodic which is similar to the scalar case. Namely, $D_{x} F$ is almost periodic if and only if it is uniformly continuous on sets of the form

Date: March 14, 2008.

2000 Mathematics Subject Classification. primary 34C27; secondary 26A24,42A75.

Key words and phrases. Almost periodic differential equation, almost periodic function, variational equation. 
$\mathbb{R} \times K$, where $K \subset \mathbb{R}^{N}$ is compact. Finally, we show that the variational equation $\dot{x}=D_{x} F(t, \mu(t)) x$ along an almost periodic solution $\mu$ is almost periodic in this case.

Notation. We denote by $\mathbb{R}^{N \times N}$ the set of all real $N \times N$ matrices. The Euclidean space $\mathbb{R}^{N}$ is equipped with the Euclidean norm $\|\cdot\|$, which is induced by the scalar product $\langle\cdot, \cdot\rangle$, where $\langle x, y\rangle:=\sum_{i=1}^{N} x_{i} y_{i}$. The $(N-1)$-sphere of the $\mathbb{R}^{N}$ is defined by $\mathbb{S}^{N-1}:=\left\{x \in \mathbb{R}^{N}:\|x\|=1\right\}$.

\section{Almost periodic functions}

Throughout the paper, we distinguish between almost periodic functions $f: \mathbb{R} \rightarrow$ $\mathbb{R}^{N}$ and functions $F: \mathbb{R} \times \mathbb{R}^{N} \rightarrow \mathbb{R}^{N}$ which are almost periodic uniformly in the second argument. The definitions are given as follows.

A function $f: \mathbb{R} \rightarrow \mathbb{R}^{N}$ is called (Bohr) almost periodic if the set

$$
\mathcal{T}(f, \varepsilon):=\{\tau \in \mathbb{R}:|f(t)-f(\tau+t)|<\varepsilon \text { for all } t \in \mathbb{R}\}
$$

is relatively dense in $\mathbb{R}$ for all $\varepsilon>0$. Note that a set $L \subset \mathbb{R}$ is relatively dense if and only there exists a $T>0$ such that $[t, t+T] \cap L \neq \emptyset$ for all $t \in \mathbb{R}$. An almost periodic function is necessarily uniformly continuous on $\mathbb{R}$ (see, e.g., [Cor68, Theorem 1.3] or [Fin74, Corollary 1.15]).

Next we consider almost periodic functions depending on a parameter $x \in \mathbb{R}^{N}$. Our studies are motivated by almost periodic differential equations $\dot{x}=F(t, x)$. We call a function $F: \mathbb{R} \times \mathbb{R}^{N} \rightarrow \mathbb{R}^{N}$ (Bohr) almost periodic uniformly in $x$ if for all compact sets $K \subset \mathbb{R}^{N}$ and $\varepsilon>0$, the set

$$
\mathcal{T}(F, \varepsilon, K):=\{\tau \in \mathbb{R}:\|F(t, x)-F(\tau+t, x)\|<\varepsilon \text { for all } t \in \mathbb{R} \text { and } x \in K\}
$$

is relatively dense in $\mathbb{R}$. A function $F$ which is almost periodic uniformly in $x$ is necessarily uniformly continuous on sets of the form $\mathbb{R} \times K$, where $K \subset \mathbb{R}^{N}$ is compact (see, e.g., [Sel67, Lemma 13]). If $F$ is a $C^{1}$-function, the derivative of $F$ with respect to $x \in \mathbb{R}^{N}$ will be denoted by $D_{x} F: \mathbb{R} \times \mathbb{R}^{N} \rightarrow \mathbb{R}^{N \times N}$.

\section{A first counterexample}

This section is devoted to the explicit construction of a continuously differentiable almost periodic function whose derivative is not almost periodic.

First of all, we define a continuous auxiliary function $h:[0,1] \rightarrow[-1,1]$ (see also Figure 1) by

$$
h(t):=\left\{\begin{array}{ccc}
4 t & : & t \in\left[0, \frac{1}{4}\right] \\
2-4 t & : & t \in\left[\frac{1}{4}, \frac{3}{4}\right] \\
4 t-4 & : & t \in\left[\frac{3}{4}, 1\right] .
\end{array}\right.
$$

Note that $\int_{0}^{1} h(t) \mathrm{d} t=0$.

Moreover, we define the continuous functions $\tilde{g}_{i}: \mathbb{R} \rightarrow[-1,1], i \in \mathbb{N}$, by

$$
\tilde{g}_{i}(t):=\left\{\begin{array}{cc}
h\left(2^{i} t+2-2^{i}\right) & : \quad t \in\left[1-\left(\frac{1}{2}\right)^{i-1}, 1-\left(\frac{1}{2}\right)^{i}\right], \\
0 & : t \notin\left[1-\left(\frac{1}{2}\right)^{i-1}, 1-\left(\frac{1}{2}\right)^{i}\right] .
\end{array}\right.
$$

$\tilde{g}_{i}$ is a contracted version of $h$ in $t$-direction, cf. Figure 2 for $i=1,2,3$. Note that the support of $\tilde{g}_{i}$ is a subset of $[0,1]$. 


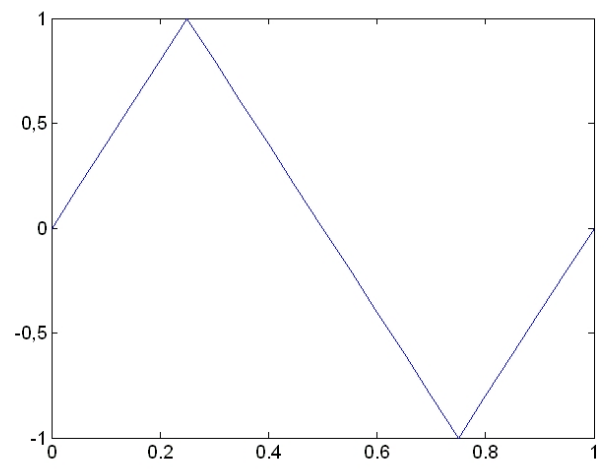

Figure 1. The function $h$.
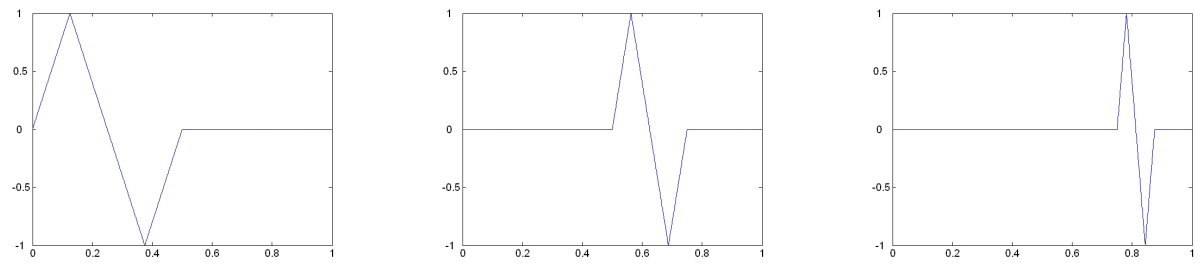

Figure 2. The functions $\tilde{g}_{1}, \tilde{g}_{2}$, and $\tilde{g}_{3}$. The peak moves to the right and is contracted in $t$-direction.

We now define $g_{i}: \mathbb{R} \rightarrow[-1,1]$ by

$$
g_{i}(t):=\sum_{k \in \mathbb{Z}} \tilde{g}_{i}\left(t+(2 k-1) 2^{i}\right) \quad \text { for all } t \in \mathbb{R} .
$$

This function is periodic with period $2^{i+1}$. The $t$-values with $g_{i}(t) \neq 0$ belong to the set $\left\{t \in \mathbb{R}: 2^{i} \mid\lfloor t\rfloor\right.$ and $\left.2^{i+1} \nmid\lfloor t\rfloor\right\}$. Note that $\int_{N}^{N+1} g_{i}(t) \mathrm{d} t=0$ for all $N \in \mathbb{Z}$.

Finally, we define $g: \mathbb{R} \rightarrow[-1,1]$ by

$$
g(t):=\sum_{i \in \mathbb{N}} g_{i}(t) \quad \text { for all } t \in \mathbb{R}
$$

(see Figure 3 ). Since the intersection of the support $\tilde{g}_{i}$ and the support of $\tilde{g}_{j}$ consists of at most one point for $i \neq j$, at most one value $g_{i}(t), i \in \mathbb{N}$, is non-zero for each $t$, and thus, the convergence of the sum in (3.1) follows. Note that $\int_{N}^{N+1} g(t) \mathrm{d} t=0$ for all $N \in \mathbb{Z}$.

The next theorem states that the integral of $g$ is almost periodic with a derivative which is not almost periodic.

Theorem 3.1. Define the integral of $g$,

$$
f(t):=\int_{0}^{t} g(s) \mathrm{d} s \text { for all } t \in \mathbb{R},
$$

with $g: \mathbb{R} \rightarrow[-1,1]$ as defined above. Then the following statements hold:

(i) $f$ is continuously differentiable, 


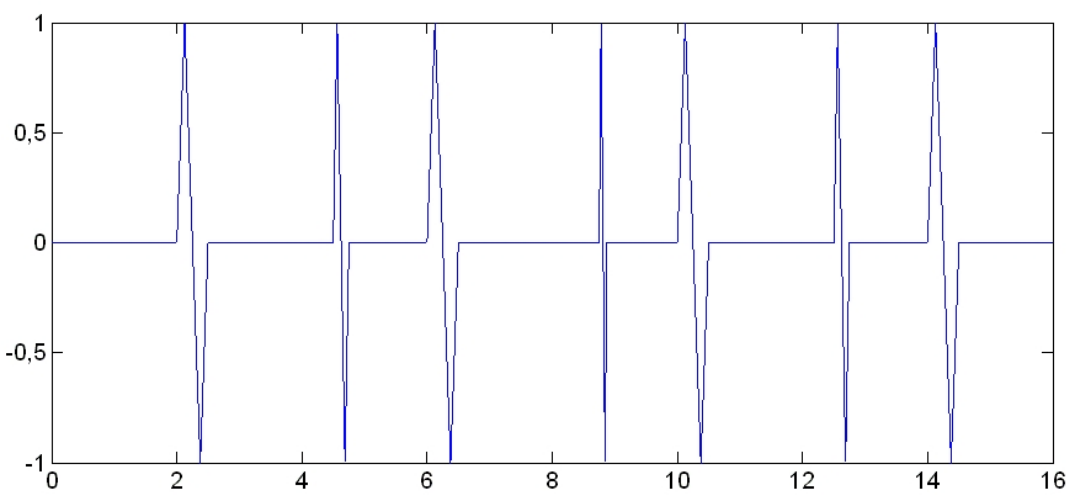

Figure 3 . The function $g$ : The wider peaks of $\tilde{g}_{1}$ occur after 2,6 , 10 and 14 , the medium peaks of $\tilde{g}_{2}$ occur after 4 and 12 , and the thin peak of $\tilde{g}_{3}$ occurs after 8 .

(ii) $f^{\prime}=g$ is not almost periodic,

(iii) $f$ is almost periodic.

Proof. Since $g$ is continuous, its integral $f$ is continuously differentiable, and assertion (i) follows immediately.

However, $f^{\prime}=g$ is not uniformly continuous, since the peaks of $\tilde{g}_{i}$ become thinner for $i \rightarrow \infty$, and due to [Cor68, Theorem 1.3], this means that $g$ is not almost periodic.

For the proof of (iii), we need the following property of the functions $\tilde{g}_{i}, i \in \mathbb{N}$. By the change of variables $s=2^{i} t+2-2^{i}$, we get

$$
\int_{0}^{\theta} \tilde{g}_{i}(t) \mathrm{d} t=\int_{1-\left(\frac{1}{2}\right)^{i-1}}^{\theta} \tilde{g}_{i}(t) \mathrm{d} t=2^{-i} \int_{0}^{2^{i} \theta+2-2^{i}} h(s) \mathrm{d} s
$$

for all $\theta \in\left[1-\left(\frac{1}{2}\right)^{i-1}, 1-\left(\frac{1}{2}\right)^{i}\right]$; for all other $\theta$, the integral is zero. Hence, using $\max _{s \in[0,1]}\left|\int_{0}^{s} h(t) \mathrm{d} t\right|=\frac{1}{4}$, we obtain

$$
\max _{\theta \in[0,1]}\left|\int_{0}^{\theta} \tilde{g}_{i}(t) \mathrm{d} t\right|=2^{-(i+2)} .
$$

It remains to show assertion (iii), the almost periodicity of $f$. Let $\varepsilon>0$ and choose $j \in \mathbb{N}$ such that $\varepsilon>2^{-(j+2)}$. In the following, we will show that the set $S:=\{\tau \in \mathbb{R}:|f(t)-f(\tau+t)|<\varepsilon$ for all $t \in \mathbb{R}\}$ is relatively dense by proving that $\left\{2^{j+1} l: l \in \mathbb{Z}\right\} \subset S$. Thus, we seek to prove that

$$
\left|f(t)-f\left(2^{j+1} l+t\right)\right|<\varepsilon \quad \text { for all } t \in \mathbb{R} \text { and } l \in \mathbb{Z} .
$$


Thereto, we choose $t \in \mathbb{R}$ and $l \in \mathbb{Z}$ arbitrarily, and we write $t=t_{0}+\theta$, where $t_{0}:=\lfloor t\rfloor \in \mathbb{Z}$ and $\theta \in[0,1)$. We obtain

$$
\begin{aligned}
f\left(2^{j+1} l+t\right)-f(t) & =\int_{t_{0}+\theta}^{2^{j+1} l+t_{0}+\theta} g(s) \mathrm{d} s \\
& =\int_{t_{0}+\theta}^{t_{0}+1} g(s) \mathrm{d} s+\underbrace{\int_{t_{0}+1}^{2^{j+1} l+t_{0}} g(s) \mathrm{d} s}_{=0}+\int_{2^{j+1} l+t_{0}}^{2^{j+1} l+t_{0}+\theta} g(s) \mathrm{d} s \\
& =-\int_{t_{0}}^{t_{0}+\theta} g(s) \mathrm{d} s+\int_{2^{j+1} l+t_{0}}^{2^{j+1} l+t_{0}+\theta} g(s) \mathrm{d} s,
\end{aligned}
$$

since $\int_{N}^{N+1} g(s) d s=0$ for all $N \in \mathbb{Z}$.

We now choose $i \in \mathbb{N}_{0}$ such that $2^{i} \mid t_{0}$ and $2^{i+1} \nmid t_{0}$ and consider the two cases $i \leq j$ and $i>j$.

Case 1. $i \leq j$.

In this case, we have $2^{i} \mid\left(t_{0}+2^{j+1} l\right)$ and $2^{i+1} \nmid\left(t_{0}+2^{j+1} l\right)$. Indeed, in contradiction to the second statement, assume that $2^{i+1} \mid\left(t_{0}+2^{j+1} l\right)$. Then, since $2^{i+1} \mid 2^{j+1} l$, this implies $2^{i+1} \mid t_{0}$, which is a contradiction. Hence, there are $k, m \in \mathbb{Z}$ which allow the representations $t_{0}=(2 k-1) 2^{i}$ and $t_{0}+2^{j+1} l=(2 m-1) 2^{i}$, and we obtain

$$
\int_{2^{j+1} l+t_{0}}^{2^{j+1} l+t_{0}+\theta} g(s) \mathrm{d} s-\int_{t_{0}}^{t_{0}+\theta} g(s) \mathrm{d} s=\int_{0}^{\theta} \tilde{g}_{i}(s) \mathrm{d} s-\int_{0}^{\theta} \tilde{g}_{i}(s) \mathrm{d} s=0 .
$$

This means $f\left(2^{j+1} l+t\right)-f(t)=0$.

Case 2. $i>j$.

We choose $p \in \mathbb{N}_{0}$ with $2^{p} \mid\left(t_{0}+2^{j+1} l\right)$ and $2^{p+1} \nmid\left(t_{0}+2^{j+1} l\right)$. We have $i \geq j+1$, thus $2^{j+1}\left|2^{i}\right|\left(t_{0}+2^{j+1} l\right)$, and this implies that $p \geq j+1$. We arrive at

$$
\begin{aligned}
\left|\int_{2^{j+1} l+t_{0}}^{2^{j+1} l+t_{0}+\theta} g(s) \mathrm{d} s-\int_{t_{0}}^{t_{0}+\theta} g(s) \mathrm{d} s\right| & =\left|\int_{0}^{\theta} \tilde{g}_{p}(s) \mathrm{d} s-\int_{0}^{\theta} \tilde{g}_{i}(s) \mathrm{d} s\right| \\
& \leq 2 \max \left(\left|\int_{0}^{\theta} \tilde{g}_{p}(s) \mathrm{d} s\right|,\left|\int_{0}^{\theta} \tilde{g}_{i}(s) \mathrm{d} s\right|\right),
\end{aligned}
$$

and by (3.2), we have $\left|\int_{0}^{\theta} \tilde{g}_{i}(s) \mathrm{d} s\right| \leq 2^{-i-2}$ for all $\theta \in[0,1)$. This implies

$$
\left|\int_{2^{j+1} l+t_{0}}^{2^{j+1} l+t_{0}+\theta} g(s) \mathrm{d} s-\int_{t_{0}}^{t_{0}+\theta} g(s) \mathrm{d} s\right| \leq \frac{1}{2} \max \left(2^{-i}, 2^{-p}\right) \leq \frac{1}{2} 2^{-(j+1)}<\varepsilon,
$$

since $i, p \geq j+1$. Finally, we have $\left|f\left(2^{j+1} l+t\right)-f(t)\right|<\varepsilon$ in this case. This finishes the proof of this theorem.

\section{A Second Counterexample}

In this section, we use the example from Section 3 to construct an example of a function $F: \mathbb{R} \times \mathbb{R}^{N} \rightarrow \mathbb{R}^{N}$ which is almost periodic uniformly in the second argument, but the derivative $D_{x} F$ does not fulfill this property.

Theorem 4.1. We define the $C^{1}$-function $F: \mathbb{R} \times \mathbb{R}^{N} \rightarrow \mathbb{R}^{N}$ by

$$
F_{j}(t, x):=f\left(t-\sum_{i=1}^{N} x_{i}\right) \quad \text { for all } j \in\{1, \ldots, N\},
$$


where $f$ is the function of Theorem 3.1. Then the following statements are fulfilled:

(i) $F$ is almost periodic uniformly in $x$,

(ii) $D_{x} F$ is not almost periodic uniformly in $x$.

Proof. To show that $F$ is almost periodic uniformly in $x$, we first note that if $\tau \in \mathbb{R}$ fulfills

$$
|f(t)-f(\tau+t)|<\varepsilon \quad \text { for all } t \in \mathbb{R},
$$

then $\tau$ also fulfills

$$
\left|f\left(t-\sum_{i=1}^{N} x_{i}\right)-f\left(\tau+t-\sum_{i=1}^{N} x_{i}\right)\right|<\varepsilon \quad \text { for all } t \in \mathbb{R} \text { and } x \in K,
$$

where $K$ is a compact set. This becomes clear by making the transformation $t \mapsto t-\sum_{i=1}^{N} x_{i}$. Hence, for a compact set $K \subset \mathbb{R}^{N}$ and $\varepsilon>0$, we have

$$
\begin{aligned}
& \{\tau \in \mathbb{R}:|f(t)-f(\tau+t)|<\varepsilon / \sqrt{N} \text { for all } t \in \mathbb{R}\} \\
\subset & \{\tau \in \mathbb{R}:\|F(t, x)-F(\tau+t, x)\|<\varepsilon \text { for all } t \in \mathbb{R} \text { and } x \in K\} .
\end{aligned}
$$

Since the first set is relatively dense by Theorem 3.1, also the latter one is relatively dense, and this implies (i).

Before proving (ii), note that we have

$$
D_{x} F(t, x)=-g\left(t-\sum_{i=1}^{N} x_{i}\right) M_{I} \quad \text { for all }(t, x) \in \mathbb{R} \times \mathbb{R}^{N},
$$

where $M_{I}=(1)_{i, j=1, \ldots, N} \in \mathbb{R}^{N \times N}$ and $g$ is the function from Section 3 .

Let us now assume that $D_{x} F$ is almost periodic uniformly in $x$. Then each entry of the matrix-valued function $D_{x} F$ is almost periodic uniformly in $x$, and that means in particular for the compact set $K=\{0\}$ and any $\varepsilon>0$, that the set

$$
\mathcal{T}(g, \varepsilon)=\{\tau \in \mathbb{R}:|g(t)-g(t+\tau)|<\varepsilon \text { for all } t \in \mathbb{R}\}
$$

is relatively dense. This implies that $g$ is almost periodic, which is a contradiction to Theorem 3.1 and finishes the proof.

\section{A NECESSARY AND SUFFICIENT CONDITION FOR ALMOST PERIODICITY}

This section is devoted to a necessary and sufficient condition for the almost periodicity of the derivative $D_{x} F$ of an almost periodic function $F: \mathbb{R} \times \mathbb{R}^{N} \rightarrow \mathbb{R}^{N}$. More precisely, we prove that $D_{x} F(t, x)$ is almost periodic uniformly in $x$ if and only if it is uniformly continuous on sets of the form $\mathbb{R} \times K$, where $K \subset \mathbb{R}^{N}$ is compact.

We also show that the variational equation $\dot{x}=D_{x} F(t, \mu(t)) x$ to an almost periodic solution $\mu$ is almost periodic in this case.

Theorem 5.1. Let the $C^{1}$-function $F: \mathbb{R} \times \mathbb{R}^{N} \rightarrow \mathbb{R}^{N}$ be almost periodic uniformly in the second argument. We suppose that $D_{x} F: \mathbb{R} \times \mathbb{R}^{N} \rightarrow \mathbb{R}^{N \times N}$ is uniformly continuous on sets of the form $\mathbb{R} \times K$, where $K \subset \mathbb{R}^{N}$ is compact. Then the function $D_{x} F$ is also almost periodic uniformly in the second argument.

Proof. We fix $i \in\{1, \ldots, N\}$ and note that it is sufficient to show that the $i$-th row of the matrix $D_{x} F$, denoted by $\operatorname{grad} F_{i}$, is an almost periodic function. For $n \in \mathbb{N}$, we define the function $\varphi_{n}^{i}: \mathbb{R} \times \mathbb{R}^{N} \times \mathbb{S}^{N-1} \rightarrow \mathbb{R}$ by

$$
\varphi_{n}^{i}(t, x, \xi):=n\left(F_{i}(t, x+\xi / n)-F_{i}(t, x)\right) .
$$

Due to the mean value theorem, we obtain the representation

$$
\varphi_{n}^{i}(t, x, \xi)=\left\langle\operatorname{grad} F_{i}\left(t, x+\theta_{n}(t, x, \xi) \xi / n\right), \xi\right\rangle
$$


where the function $\theta_{n}: \mathbb{R} \times \mathbb{R}^{N} \times \mathbb{S}^{N-1} \rightarrow \mathbb{R}$ fulfills $0 \leq \theta_{n}(t, x, \xi) \leq 1$. Now choose a compact set $K \subset \mathbb{R}^{N}$ and $\varepsilon>0$. The uniform continuity of $D_{x} F$ implies that there exists an $N_{0}>0$ with

$$
\left\|\operatorname{grad} F_{i}\left(t, x+\theta_{n}(t, x, \xi) \xi / n\right)-\operatorname{grad} F_{i}(t, x)\right\|<\frac{\varepsilon}{4}
$$

for all $t \in \mathbb{R}, x \in K, \xi \in \mathbb{S}^{N-1}$ and $n \geq N_{0}$ (note that $0 \leq \theta_{n}(t, x, \xi) \leq 1$ ). Due to the Cauchy-Schwarz inequality, we obtain

$$
\left|\varphi_{n}^{i}(t, x, \xi)-\left\langle\operatorname{grad} F_{i}(t, x), \xi\right\rangle\right|<\frac{\varepsilon}{4}
$$

for all $t \in \mathbb{R}, x \in K, \xi \in \mathbb{S}^{N-1}$ and $n \geq N_{0}$. We define the compact set

$$
K^{\prime}:=\left\{x+\xi / N_{0}: x \in K,\|\xi\| \leq 1\right\}
$$

and let $\tau^{*} \in \mathcal{T}\left(F_{i}, \frac{\varepsilon}{4 N_{0}}, K^{\prime}\right)$, where

$$
\mathcal{T}\left(F_{i}, \frac{\varepsilon}{4 N_{0}}, K^{\prime}\right)=\left\{\tau \in \mathbb{R}:\left|F_{i}(t, x)-F_{i}(t+\tau, x)\right|<\frac{\varepsilon}{4 N_{0}} \text { for } t \in \mathbb{R} \text { and } x \in K^{\prime}\right\} .
$$

Note that the set $\mathcal{T}\left(F_{i}, \frac{\varepsilon}{4 N_{0}}, K^{\prime}\right)$ is relatively dense, since $F_{i}$ is almost periodic. Then we have for all $x \in K$ and $\xi \in \mathbb{S}^{N-1}$

$$
\begin{aligned}
& \left|\varphi_{N_{0}}^{i}(t, x, \xi)-\varphi_{N_{0}}^{i}\left(t+\tau^{*}, x, \xi\right)\right| \\
\leq & N_{0}\left(\left|F_{i}\left(t, x+\frac{\xi}{N_{0}}\right)-F_{i}\left(t+\tau^{*}, x+\frac{\xi}{N_{0}}\right)\right|+\left|F_{i}(t, x)-F_{i}\left(t+\tau^{*}, x\right)\right|\right) \\
< & \frac{\varepsilon}{2}
\end{aligned}
$$

Hence, we obtain for all $x \in K$ that

$$
\begin{aligned}
& \left\|\operatorname{grad} F_{i}(t, x)-\operatorname{grad} F_{i}\left(t+\tau^{*}, x\right)\right\| \\
& =\sup _{\xi \in \mathbb{S}^{N-1}}\left\langle\operatorname{grad} F_{i}(t, x)-\operatorname{grad} F_{i}\left(t+\tau^{*}, x\right), \xi\right\rangle \\
& \leq \sup _{\xi \in \mathbb{S}^{N-1}}\left|\left\langle\operatorname{grad} F_{i}(t, x), \xi\right\rangle-\varphi_{N_{0}}^{i}(t, x, \xi)\right| \\
& \quad+\sup _{\xi \in \mathbb{S}^{N-1}}\left|\varphi_{N_{0}}^{i}(t, x, \xi)-\varphi_{N_{0}}^{i}\left(t+\tau^{*}, x, \xi\right)\right| \\
& \quad+\sup _{\xi \in \mathbb{S}^{N-1}}\left|\varphi_{N_{0}}^{i}\left(t+\tau^{*}, x, \xi\right)-\left\langle\operatorname{grad} F_{i}\left(t+\tau^{*}, x\right), \xi\right\rangle\right| \\
& \text { (5.1),(5.2) }_{<}^{\frac{\varepsilon}{4}}+\frac{\varepsilon}{2}+\frac{\varepsilon}{4}=\varepsilon,
\end{aligned}
$$

and this implies that $\mathcal{T}\left(F_{i}, \frac{\varepsilon}{4 N_{0}}, K^{\prime}\right) \subset \mathcal{T}\left(\operatorname{grad} F_{i}, \varepsilon, K\right)$. Hence, the set on the right-hand side is relatively dense. Thus, $\operatorname{grad} F_{i}$ is almost periodic, and this also means that $D_{x} F$ is almost periodic, since $i$ has been chosen arbitrarily.

This theorem, together with [Sel67, Lemma 13]), implies the following sufficient and necessary condition for the almost periodicity of $D_{x} F$.

Corollary 5.1. Let the $C^{1}$-function $F: \mathbb{R} \times \mathbb{R}^{N} \rightarrow \mathbb{R}^{N}$ be almost periodic uniformly in $x$. Then $D_{x} F: \mathbb{R} \times \mathbb{R}^{N} \rightarrow \mathbb{R}^{N \times N}$ is uniformly continuous on sets of the form $\mathbb{R} \times K$, where $K \subset \mathbb{R}^{N}$ is compact, if and only if the function $D_{x} F$ is almost periodic uniformly in $x$.

Finally, we apply the results to variational equations $\dot{x}=D_{x} F(t, \mu(t)) x$, where the solution $\mu$ is supposed to be almost periodic. 
Corollary 5.2. Let the $C^{1}$-function $F: \mathbb{R} \times \mathbb{R}^{N} \rightarrow \mathbb{R}^{N}$ be almost periodic uniformly in $x$ and $D_{x} F: \mathbb{R} \times \mathbb{R}^{N} \rightarrow \mathbb{R}^{N \times N}$ be uniformly continuous on sets of the form $\mathbb{R} \times K$, where $K \subset \mathbb{R}^{N}$ is compact. Then, given an almost periodic solution $\mu: \mathbb{R} \rightarrow \mathbb{R}^{N}$ of the differential equation

$$
\dot{x}=F(t, x),
$$

the variational equation along the solution $\mu$, given by

$$
\dot{x}=D_{x} F(t, \mu(t)) x,
$$

is almost periodic (uniformly in $x$ ).

Proof. This follows from Theorem 5.1 and [Fin74, Theorem 2.11].

\section{REFERENCES}

[Cor68] C. Corduneanu, Almost Periodic Functions, Interscience Tracts in Pure and Applied Mathematics, no. 22, Interscience Publishers, New York, 1968.

[Fin74] A. M. Fink, Almost Periodic Differential Equations, Springer Lecture Notes in Mathematics, vol. 377, Springer, Berlin, Heidelberg, 1974.

[GR] P. Giesl and M. Rasmussen, Borg's criterion for almost periodic differential equations, to appear in: Nonlinear Analysis. Theory, Methods \& Applications.

[Sel67] G. R. Sell, Nonautonomous differential equations and dynamical systems - I. The basic theory, Transactions of the American Mathematical Society 127 (1967), 241-262.

Peter Giesl, Department of Mathematics, Mantell Building, University of Sussex, FALMER, BN1 9RF, UK

E-mail address: p.a.giesl@sussex.ac.uk

Martin Rasmussen ${ }^{1}$, Department of Mathematics, University of Augsburg, D-86135 Augsburg, Germany

E-mail address: martin.rasmussen@math.uni-augsburg.de

\footnotetext{
${ }^{1}$ Supported by the Bayerisches Eliteförderungsgesetz of the State of Bavaria, Germany.
} 